\title{
Diseño de una escala para la evaluación de calidad metodológica de estudios de pronóstico*
}

\author{
Drs. HÉCTOR LOSADA M. ${ }^{1,2}$, CARLOS MANTEROLA D. ${ }^{1,2}$, VIVIANA PINEDA N. ${ }^{1,2}$, \\ MANUEL VIAL G. ${ }^{1,2}$, ANTONIO SANHUEZA R. ${ }^{1}$ GRUPO MINCIR \\ Departamento de Cirugía y Traumatología, Universidad de La Frontera. \\ 2 Servicio de Cirugía, Hospital Hernán Henríquez Aravena. Temuco, Chile.
}

\begin{abstract}
Design of a methodological quality scale for prognostic studies

Background: One of the main classification systems to describe the quality of scientific publications is the level of evidence. However, for our research team, the methodological quality is multidimensional. Aim: To design a scale to determine the methodological quality of studies dealing with prognosis. Material and Method: On a first stage, five professionals with experience in research were interviewed about the items that, in their opinion, must be considered to assess the methodological quality of a manuscript. On a second stage, 121 prognostic studies were critically reviewed by four professionals with research experience In addition, the number of publications of each author was determined and the evidence level of each article was described according to the Oxford Centre for Evidence-based Medicine. A subsequent factorial analysis was performed and the fields and items of the scale were generated. Results: Sixty six percent of reviewed articles had a cohort design (prospective or retrospective), $45 \%$ were located at an evidence level $1 \mathrm{~b}$ and $39 \%$ are at an evidence level of 4 . After reviewing the articles, a factorial analysis was performed choosing an orthogonal matrix. A scale (MinCir Pr) was generated with a total of 25 items, 6 fields and a Cronbach coefficient alpha of 0.89 . Conclusions: A scale to evaluate the methodological quality of prognosis studies (MinCir Pr) was designed, fulfilling rigorous methodological steps and the criterion of multidimensionality for methodological quality.
\end{abstract}

Key words: Prognosis articles, scale design, methodological quality.

\section{Resumen}

Introducción: Uno de los principales sistemas para calificar la calidad de las publicaciones científicas ha sido los niveles de evidencia. Para nuestro equipo de investigación el constructo calidad metodológica es un constructo multidimensional. Nuestro objetivo es diseñar una escala para determinar la calidad metodológica de los estudios referentes a pronóstico. Material y Método: Diseño de generación de escalas. En la primera etapa las fuentes de los ítems fueron entrevistas en profundidad a 5 profesionales con experiencia en investigación y la revisión de la literatura de los diferentes artículos acerca de evaluación de

\footnotetext{
Financiado por proyecto DIDUFRO N¹20629.

Correspondencia: Dr. Héctor Losada M.

Casilla 54-D. Temuco, Chile

E-mail: hlosada@ufro.cl
}

*Recibido el 29 de Septiembre de 2007 y aceptado para publicación el 10 de Julio de 2008. 
calidad metodológica de los estudios de pronóstico. En la segunda etapa se revisaron 121 estudios de pronóstico por 4 profesionales con experiencia en investigación. Además se analizó el número de publicaciones del autor y se calificó el nivel de evidencia de cada artículo según el centro de evidencia de Oxford. Posteriormente se realizó el análisis factorial y la generación de dominios e ítems de la escala. Resultados: Luego de le etapa de generación de ítems, se analizaron 120 artículos referentes a pronóstico. Un $66 \%$ de los artículos tienen diseño de cohorte (prospectiva o retrospectiva); un $45 \%$ son nivel de evidencia $1 \mathrm{~b}$ y $39 \%$ son nivel de evidencia 4. Luego de la revisión de artículos se efectuó el análisis factorial escogiendo una matriz ortogonal. Se generó una escala (MinCir Pr) con un total de 25 items, 6 dominios y un coeficiente de alfa de Cronbach de 0,89. Conclusiones: Se ha diseñado una escala para la evaluación de calidad metodológica de estudios de pronóstico (MinCir Pr) cumpliendo rigurosos pasos metodológicos y el criterio de multidimensionalidad de la calidad metodológica.

Palabras clave: Estudios de pronóstico, diseño de escala, calidad metodológica.

\section{Introducción}

Durante muchos años las decisiones en salud se tomaron basadas en la experiencia de las personas. La medicina basada en evidencias (MBE) se inicia como una disciplina para mejorar la calidad de las decisiones en salud. La MBE tiene como objeto sistematizar la forma de obtener el mejor conocimiento médico.

Uno de los aspectos más importantes de MBE es la evaluación de la calidad metodológica de los diferentes estudios. Uno de los principales sistemas para calificar la calidad de las publicaciones científicas ha sido los niveles de evidencia ${ }^{1}$, que evalúa aspectos como el diseño de investigación, el riesgo de sesgos y la homogeneidad entre otros.

Existen guías para evaluación de diversos tipos de estudios realizadas por Evidence-Based Medicine Working Group y publicados en Journal of American Medical Association (JAMA), que se han convertido en prueba de referencia o "gold standard" para evaluar calidad metodológica, éstas evalúan diversos aspectos sin puntuación numérica ${ }^{2-31}$. Recientemente, han aparecido recomendaciones para el reporte de estudios con diferente diseño: CONSORT (Consolidated Standarts of Reporting Trials) para ensayos clínicos ${ }^{32}$ y STARD (Standarts for Reporting of Diagnostic Accuracy) para estudios de diagnóstico ${ }^{33}$; y se han utilizado estas recomendaciones como puntuaciones de evaluación de calidad metodológica. Sin embargo, éstas no fueron diseñadas para evaluación de calidad metodológica y no han sido validadas para tal fin.

Para nuestro equipo de investigación el constructo calidad metodológica es un constructo multidimensional. En ésta línea nuestro equipo diseñó un escore para comparar la calidad metodológica de estudios de terapia con diferente diseño de investigación que podría ser utilizado en revisiones sistemáticas. Este escore consta de 3 principales dominios: tipo de diseño de investigación, tamaño de la población a estudio con un ajuste si existe una justificación del tamaño de la muestra y metodología empleada ${ }^{34}$.

Sin embargo, este escore no aplica al momento de evaluar estudios de pronóstico, diagnóstico, económicos, etc.

Los estudios de pronóstico son cada vez más importantes en el mundo quirúrgico. Para los estudios de pronóstico el diseño de cohorte sería considerado "el mejor nivel de evidencia". Sin embargo, la conducción de un estudio de cohorte plantea un desafío para el mundo de la epidemiología clínica por múltiples factores, entre ellos la definición de la exposición, el tiempo y la calidad del seguimiento de los grupos y el costo.

Nuestra hipótesis es que existen ítems y dominios que permiten diseñar una escala para evaluación de calidad metodológica en estudios de pronóstico. Nuestro objetivo es diseñar una escala para determinar la calidad metodológica de los estudios referentes a pronóstico; que nos permite una comparación de estudios con diferentes diseños y metodologías, de forma tal de poder realizar RSL en el ámbito del pronóstico, para poder de esta forma dar respuesta a interrogantes puntuales con un adecuado nivel de evidencia.

\section{Material y Método}

Diseño: Generación de escala. Este diseño tiene varias etapas:

La primera etapa es la búsqueda de los diferentes ítems para la construcción de la escala (primer borrador). En ésta se utilizó metodología de investigación cualitativa.

La segunda etapa se inicia con una aplicación del primer borrador de la escala, para luego realizar en análisis factorial. El análisis factorial es una téc- 
nica de reducción de dimensionalidad de los datos; su propósito último es buscar el número mínimo de dimensiones capaces de explicar el máximo de información contenida en los datos. En éste etapa se escogen los dominios de la escala y se realiza el análisis de confiabilidad (alfa de Cronbach).

Población: En la primera etapa de la escala las fuentes de los ítems fueron entrevistas en profundidad a 5 profesionales con experiencia en investigación y la revisión de la literatura de los diferentes artículos acerca de evaluación de calidad metodológica ${ }^{1-37}$.

En la segunda etapa la población fueron 121 estudios de pronóstico que fueron revisados por 4 profesionales con experiencia en investigación, diferentes a los participantes en la primera etapa. Además se analizó el número de publicaciones del autor y se calificó el nivel de evidencia de cada artículo según el centro de evidencia de Oxford ${ }^{1}$.

Tamaño de la muestra: Para el tamaño de muestra en los estudios de generación de escalas existen varias recomendaciones. Una de estas hace mención a un intervalo de 5 a 10 mediciones por cada ítem de la escala ${ }^{38}$.

El proceso matemático del análisis factorial y la rotación de matrices requiere un mínimo de $120 \mathrm{me}$ diciones. Es por esto que elegimos este número de $\operatorname{artículos}^{39}$.

Selección de artículos: Los criterios de inclusión para los diferentes artículos fueron:

- Estudios registrados en la base de datos Medline, bajo el término libre "prognosis study".

- No habrá restricción en el año, ni idioma de publicación.

De los artículos inicialmente seleccionados se revisará el resumen y se excluirán los artículos no relacionados con pronóstico. Posteriormente, se buscarán los artículos en texto completo para su revisión.

Selección de investigadores: Los criterios de inclusión para los investigadores participantes fueron:

- Certificación de epidemiólogo clínico o bioestadístico.

- Pertenecer a una unidad o centro de epidemiología clínica.

- Aceptación de participación en el protocolo.

Plan de análisis: Se utilizó metodología de investigación cualitativa en la primera etapa para el análisis de las entrevistas en profundidad. Este se realizó con el programa Atlas-Ti®.
Se utilizó estadística descriptiva, con cálculo de medidas de tendencia central, dispersión y tendencia extrema; análisis divariado utilizando $\chi^{2}$ de Pearson o test exacto de Fischer para variables categóricas y t-test, ANOVA y Kruskal Wallis para variables continuas. Los datos fueron analizados utilizando el programa Stata ${ }^{\circledR}$ 8.0.

Aspectos éticos y de financiamiento: Este protocolo fue financiado por el proyecto DIUFRO No 120629 de la Dirección de Investigación y Desarrollo de la Universidad de La Frontera.

\section{Resultados}

La primera etapa se enfocó a la selección de posibles ítems de la escala. Las fuentes de ítems fueron las entrevistas en profundidad a 5 profesionales con experiencia en investigación y la revisión de la literatura de los diferentes artículos acerca de evaluación de calidad metodológica de los estudios de pronóstico.

Después de obtener los ítems de éstas 2 fuentes (metodología cualitativa y la revisión de la literatura) se realizó una reunión de consenso del equipo de investigación para realizar un análisis de los diferentes ítems encontrados; tratando de excluir aquellos que evaluaran los mismos aspectos $\mathrm{y}$ optimizando la traducción al castellano (Tabla 1).

Posteriormente, se procedió a buscar los artículos en texto completo, para lo cual se tuvo la colaboración de la Universidad de Emory (E.U.).

Se contó para la lectura completa con 130 artículos de los cuales se excluyeron $9^{40-170}$. Las causas de exclusión fueron:

- Tres (3) artículos eran ensayos clínicos aleatorios que evaluaban terapia.

- Tres (3) eran revisiones sistemáticas de artículos de terapia.

- Dos (2) eran estudios de metodología cualitativa que no hacían evaluación de pronóstico en ninguno de sus aspectos.

- 1 (un) estudio era una prueba diagnóstica.

En resumen, en esta primera etapa se analizaron 121 artículos relacionados con pronóstico, sin importar el diseño de investigación.

Los artículos analizados tienen las siguientes características:

- 56 artículos $(46 \%)$ no tienen hipótesis.

- 6 artículos $(5 \%)$ no tienen objetivos.

- 83 artículos (68\%) no mencionan el diseño de investigación utilizado.

- 118 artículos (97\%) no poseen cálculo del tamaño de la muestra. 
Tabla 1. Ítems obtenidos de metodología cualitativa y revisión de la literatura

- Se identifica una hipótesis claramente

- Se identifica un objetivo claramente

- Es un estudio de cohorte

- Se enuncia claramente el diseño del estudio

- Se describe la población del estudio

- Hay cálculo del tamaño de la muestra

- Se enuncia el método de muestreo

- Se justifica el tamaño de la muestra

- Se define un período de reclutamiento de la muestra

- Se define el lugar de reclutamiento de la muestra

- Se describen los criterios de inclusión

- Se definen los criterios de exclusión

- Existe coherencia entre la población y el objetivo

- Se enuncia el porcentaje de pérdida de seguimiento

- Se describen las características de los sujetos perdidos

- Se hicieron esfuerzos por recolectar la información de los sujetos perdidos

- Las causas de la pérdida del seguimiento son descritas

- Se analizaron las diferencias entre las características de los sujetos con pérdida de seguimiento y los que no

- Se define la variable de exposición

- El método para medir la variable de exposición es válido y confiable

- Se define las unidades de medida de la variable de exposición

- Una adecuada proporción de la muestra tiene datos sobre la variable de exposición

- El método y las unidades de medición de la variable de exposición son los mismos para todos los sujetos

- Se define la variable de desenlace o respuesta

- El método para medir la variable de desenlace es válido y confiable

- Se define las unidades de medida de la variable de desenlace

- Una adecuada proporción de la muestra tiene datos completos sobre la variable de desenlace

- El método y las unidades de medición de la variable de desenlace son los mismos para todos los sujetos

- El seguimiento en el tiempo está definido

- El tiempo de seguimiento es suficiente para la aparición de la variable de desenlace

- Considera variables de confusión

- Se definen adecuadamente las variables de confusión

- Los métodos de medida de las variables de confusión son válidos y confiables

- Con los datos presentados en el estudio es posible determinar medidas de asociación y riesgo

- Se realizan modelos predictivos o de asociación
- 24 artículos (20\%) no tienen criterios de inclusión.

- 64 artículos (52\%) no tienen criterios de exclusión.

- 24 artículos $(20 \%)$ no tienen una adecuada definición de la variable de exposición.

- 6 artículos (5\%) no tienen definición de la variable de respuesta.

- 84 artículos (69\%) no consideran variables de confusión.

- Con los datos presentados en 46 artículos (38\%) no es posible calcular las medidas de riesgo o asociación.

- 38 artículos (31\%) no consideran modelos predictivos o de asociación.

Los diseños de investigación de los estudios se describen en la Tabla 2.

Los niveles de evidencia de los estudios se describen en la Tabla 3.

El promedio de artículos publicados por el autor fue de $36,56 \pm 47,93$ con una mediana de 17,5 y valores extremos de 1 y 309 .

$\mathrm{Si}$ analizamos a los autores de los artículos con nivel de evidencia 1 tenemos que el promedio de las publicaciones fue de 44,59 \pm 57,30 en comparación con los autores de los artículos con nivel de

Tabla 2. Descripción de diseños de investigación

\begin{tabular}{lrc}
\hline Diseño & n & \% \\
\hline Meta-análisis & 1 & 0,82 \\
Revisión sistemática de la literatura & 1 & 0,82 \\
Cohorte prospectiva & 53 & 43,44 \\
Cohorte retrospectiva & 28 & 22,95 \\
Corte transversal & 1 & 0,82 \\
Series de casos & 38 & 31,15 \\
Total & 122 & 100 \\
\hline
\end{tabular}

Tabla 3. Descripción de niveles de evidencia

\begin{tabular}{crr}
\hline Nivel de evidencia & n & \multicolumn{1}{c}{$\%$} \\
\hline $1 \mathrm{a}$ & 1 & 0,82 \\
$1 \mathrm{~b}$ & 55 & 45,08 \\
$2 \mathrm{a}$ & 3 & 2,46 \\
$2 \mathrm{~b}$ & 14 & 11,48 \\
$2 \mathrm{c}$ & 1 & 0,82 \\
4 & 48 & 39,34 \\
\hline
\end{tabular}


evidencia 2, y 4 cuyo promedio de publicaciones fue $27,86 \pm 33,26$; sin embargo, esta diferencia no es estadísticamente significativa $(\mathrm{p}=0,0752)$.

Se encontró asociación entre el diseño empleado en los estudios y la calificación de los niveles de evidencia $(\mathrm{p}=000)$; con un coeficiente de correlación de 0,6607.

Posteriormente, se realizó el análisis factorial donde se escogió una matriz ortogonal para la rotación de los factores. Con base en éste análisis se extrajo el número mínimo de dimensiones capaces de explicar el máximo de información contenida en los datos.

Así tenemos los siguientes dominios e ítems:

\section{Dominio 1}

- Se define la variable de desenlace.

- El método para medir la variable de desenlace es válido y confiable.

- Se define las unidades de medida de la variable de desenlace.

- Una adecuada proporción de la muestra tiene datos completos de la variable de desenlace.

- El método y las unidades de medición de la/las variables de desenlace son los mismos para todos los pacientes.

\section{Dominio 2}

- Se define la variable de exposición.

- El método para medir la variable de exposición es válido y confiable.

- Se define las unidades de medida de la variable de exposición.

- Una adecuada proporción de la muestra tiene datos completos de la variable de exposición.

- El método y las unidades de medición de la/las variables de exposición son los mismos para todos los pacientes.

\section{Dominio 3}

- Se enuncia el porcentaje de pérdida de seguimiento.

- Se describen las características de los sujetos perdidos.

- Se hicieron esfuerzos por recolectar la información de los sujetos perdidos.

- Las causas de pérdida de seguimiento son descritas.

- Se analizaron las diferencias entre las características de los sujetos con pérdida de seguimiento y los que no.

\section{Dominio 4}

- Con los datos presentados es posible determinar las medidas de riesgo y asociación.

- Se realizan modelos de asociación o predictivos.
- El modelo elegido es adecuado según el diseño del estudio.

- Las conclusiones del estudio son coherentes con el objetivo y la metodología empleadas.

\section{Dominio 5}

- Se enuncia claramente el diseño del estudio.

- Considera variables de confusión.

- Se definen adecuadamente las variables de confusión.

- Los métodos para medir las variables de confusión son válidos y confiables.

\section{Dominio 6}

- Hay cálculo del tamaño de la muestra.

- Se justifica el tamaño de la muestra.

El resultado del cálculo del alfa de Cronbach es: Dominio 1: alfa de Cronbach de 0,95.

Dominio 2: alfa de Cronbach de 0,95.

Dominio 3: alfa de Cronbach de 0,88.

Dominio 4: alfa de Cronbach de 0,82.

Dominio 5: alfa de Cronbach de 0,75.

Dominio 6: alfa de Cronbach de 0,94.

Con esto tenemos una escala (MinCir Pr) con un total de 25 ítems, 6 dominios y un coeficiente de alfa de Cronbach de 0,89.

Posteriormente, se decidió utilizar una puntuación dicotómica para la escala, utilizando 1 para el ítem que no fue cumplido por el artículo y 5 para el ítem que fue cumplido por el artículo.

La escala (MinCir Pr) se describe en la Tabla 4.

\section{Discusión}

Hay un creciente número de revisiones sistemáticas que están siendo publicadas en las diferentes disciplinas médicas. Esto tiene la ventaja de contar con la información contenida en varios estudios del mismo tema de investigación.

Sin embargo varios artículos se han referido a la inconsistente calidad de algunas revisiones sistemáticas ${ }^{171,172}$ y por tanto los errores de las conclusiones extraídas de éstas; que en algunas circunstancias regulan el quehacer médico.

Dentro de los aspectos más importantes está la heterogeneidad y calidad de los estudios incluidos. Esta escala podría colaborar a resolver en parte éste problema al permitir evaluar numéricamente la calidad de los diferentes estudios e incluso poder sacar promedios ponderados por la calidad de cada trabajo de las diferentes variables, técnica utilizada por el equipo de trabajo en algunas de sus revisiones sistemáticas. 
Tabla 4. Escala MinCir para estudios de pronóstico (MinCir Pr)

\section{Escala MINCIR pronóstico}

\section{Dominio 1 (Variable de desenlace)}

Se define la variable de desenlace

El método para medir la variable de desenlace es válido y confiable

Se define las unidades de medida de la variable de desenlace

Una adecuada proporción de la muestra tiene datos completos de la variable de desenlace

El método y las unidades de medición de la/las variables de desenlace son los mismos para todos los pacientes

\section{Dominio 2 (Variable de exposición)}

Se define la variable de exposición

El método para medir la variable de exposición es válido y confiable

Se define las unidades de medida de la variable de exposición

Una adecuada proporción de la muestra tiene datos completos de la variable de exposición

El método y las unidades de medición de la/las variables de exposición son los mismos para todos los pacientes

\section{Dominio 3 (Seguimiento)}

Se enuncia el porcentaje de pérdida de seguimiento

Se describen las características de los sujetos perdidos

Se hicieron esfuerzos por recolectar la información de los sujetos perdidos

Las causas de pérdida de seguimiento son descritas

Se analizaron las diferencias entre las características de los sujetos con pérdida de seguimiento y los que no

\section{Dominio 4 (Análisis y conclusiones)}

Con los datos presentados es posible determinar las medidas de riesgo y asociación

Se realizan modelos de asociación o predictivos

El modelo elegido es adecuado según el diseño del estudio

Las conclusiones del estudio son coherentes con el objetivo y la metodología empleadas

\section{Dominio 5 (Diseño y variables de confusión)}

Se enuncia claramente el diseño del estudio

Considera variables de confusión

Se definen adecuadamente las variables de confusión

Los métodos para medir las variables de confusión son válidos y confiables

\section{Dominio 6 (Tamaño de la muestra)}

Hay cálculo del tamaño de la muestra

Se justifica el tamaño de la muestra

Total 
Con respecto a la metodología de la escala, ésta cumplió varios pasos importantes en su proceso de diseño:

- Extracción de posibles ítems con base en la experiencia de los autores y la literatura.

- Revisión de los posibles ítems por un comité de expertos tratando de excluir aquellos que evaluaran los mismos aspectos y optimizando la traducción al castellano.

- Aplicación del cuestionario inicial a 120 artículos de pronóstico.

- Realización de análisis factorial para extraer ítems y dominios.

- Cálculo de la consistencia interna.

En cuanto a las descripción de los estudios incluidos llama la atención algunas cifras como el $68 \%$ de estudios que no mencionan el diseño de investigación, el $97 \%$ que no poseen cálculo del tamaño de la muestra, el $20 \%$ que no tienen criterio de inclusión y el $52 \%$ sin criterio de exclusión. Esto podría interpretarse como una baja calidad en la metodología empleada y llama la atención a los revisores y editores de las diferentes revistas científicas a mejorar sus estándares de publicación. Además esto puede reflejar una débil formación académica en metodología de investigación, lo cual es una realidad en Latinoamérica y motiva a diversos centros a diseñar programas para entrenamiento en éste aspecto.

Un aspecto adicional que se midió fue el número de publicaciones del autor de cada artículo. En este aspecto no encontramos asociación significativamente estadística entre el promedio de artículos publicados de los autores con artículos nivel de evidencia 1 y los autores con artículos nivel de evidencia 2 y $4(p=0,0752)$. Esto puede ser explicado por algún sesgo en el número de publicaciones, ya que en muchos trabajos los autores son residentes o fellows y el staff o investigador principal no aparece como autor. Además cada vez hay un número creciente de publicaciones que no están indexadas en MEDLINE, que son muy importantes para las diferentes sociedades científicas donde publican investigadores de reconocida calidad.

Además, ésta nueva escala cumple el criterio teórico de multidimensionalidad de la calidad metodológica, aspecto muy importante para nuestro grupo de investigación. La siguiente etapa será la validación de la escala.

\section{Conclusión}

Se ha diseñado una escala para la evaluación de calidad metodológica de estudios de pronóstico
(MinCir Pr) cumpliendo el criterio de multidimensionalidad de la calidad metodológica. Una vez validada podría ser utilizada en revisiones sistemáticas.

\section{Referencias}

1. Oxford Centre for Evidence-based Medicine. Levels of evidence. May 2001. Produced by Phillips B, Ball C, Sackett D, et al., since November 1998. Disponible en: http://163.1.96.10/docs/levels.html\#levels. Consultado junio 25 de 2005.

2. Guyatt GH, Haynes RB, Jaeschke RZ, Cook DJ, Green L, Naylor CD, et al. Users' Guides to the Medical Literature: XXV. Evidence-based medicine: principles for applying the Users' Guides to patient care. Evidence-Based Medicine Working Group. JAMA 2000; 284: 1290-1296.

3. Richardson WS, Wilson MC, Williams JW Jr, Moyer VA, Naylor CD. Users' guides to the medical literature: XXIV. How to use an article on the clinical manifestations of disease. Evidence-Based Medicine Working Group. JAMA 2000; 284: 869-875.

4. Giacomini MK Cook DJ. Users' guides to the medical literature: XXIII. Qualitative research in health care B. What are the results and how do they help me care for my patients? Evidence-Based Medicine Working Group. JAMA 2000; 284: 478-482.

5. Giacomini MK, Cook DJ. Users' guides to the medical literature: XXIII. Qualitative research in health care A. Are the results of the study valid? Evidence-Based Medicine Working Group. JAMA 2000; 284: 357 362 .

6. McGinn TG, Guyatt GH, Wyer PC, Naylor CD, Stiell IG, Richardson WS. Users' guides to the medical literature: XXII: how to use articles about clinical decision rules. Evidence-Based Medicine Working Group. JAMA 2000; 284: 79-84.

7. McAlister FA, Straus SE, Guyatt GH, Haynes RB. Users' guides to the medical literature: XX. Integrating research evidence with the care of the individual patient. Evidence-Based Medicine Working Group. JAMA 2000; 283: 2829-2836.

8. Hunt DL, Jaeschke R, McKibbon KA. Users' guides to the medical literature: XXI. Using electronic health information resources in evidence-based practice. Evidence-Based Medicine Working Group. JAMA 2000; 283: 1875-1879.

9. Bucher HC, Guyatt GH, Cook DJ, Holbrook A, McAlister FA. Users' guides to the medical literature: XIX. Applying clinical trial results. A. How to use an article measuring the effect of an intervention on surrogate end points. Evidence-Based Medicine Working Group. JAMA 1999; 282: 771-778.

10. Barratt A, Irwig L, Glasziou P, Cumming RG, Raffle 
A, Hicks $\mathrm{N}$ et al. Users' guides to the medical literature: XVII. How to use guidelines and recommendations about screening. Evidence-Based Medicine Working Group. JAMA 1999; 281: 2029-2034.

11. Guyatt GH, Sinclair J, Cook DJ, Glasziou P. Users' guides to the medical literature: XVI. How to use a treatment recommendation. Evidence-Based Medicine Working Group and the Cochrane Applicability Methods Working Group. JAMA 1999; 281: 18361843.

12. Richardson WS, Wilson MC, Guyatt GH, Cook DJ, Nishikawa J. Users' guides to the medical literature: $\mathrm{XV}$. How to use an article about disease probability for differential diagnosis. Evidence-Based Medicine Working Group. JAMA 1999; 281: 1214-1219.

13. Dans AL, Dans LF, Guyatt GH, Richardson S. Users' guides to the medical literature: XIV. How to decide on the applicability of clinical trial results to your patient. Evidence-Based Medicine Working Group. JAMA 1998; 279: 545-549.

14. O'Brien BJ, Heyland D, Richardson WS, Levine M, Drummond MF. Users' guides to the medical literature. XIII. How to use an article on economic analysis of clinical practice. B. What are the results and will they help me in caring for my patients? Evidence-Based Medicine Working Group. JAMA 1997; 277: $1802-$ 1806.

15. Drummond MF, Richardson WS, O'Brien BJ, Levine $\mathrm{M}$, Heyland D. Users' guides to the medical literature. XIII. How to use an article on economic analysis of clinical practice. A. Are the results of the study valid? Evidence-Based Medicine Working Group. JAMA 1997; 277: 1552-1557.

16. Guyatt GH, Naylor CD, Juniper E, Heyland DK, Jaeschke R, Cook DJ. Users' guides to the medical literature. XII. How to use articles about health-related quality of life. Evidence-Based Medicine Working Group. JAMA 1997; 277: 1232-1237.

17. Naylor CD, Guyatt GH. Users' guides to the medical literature. XI. How to use an article about a clinical utilization review. Evidence-Based Medicine Working Group. JAMA 1996; 275: 1435-1439.

18. Naylor CD, Guyatt GH. Users' guides to the medical literature. X. How to use an article reporting variations in the outcomes of health services. The EvidenceBased Medicine Working Group. JAMA 1996; 275: 554-558.

19. Guyatt GH, Sackett DL, Sinclair JC, Hayward R, Cook DJ, Cook RJ. Users' guides to the medical literature. IX. A method for grading health care recommendations. Evidence-Based Medicine Working Group. JAMA 1995; 274: 1800-1804.

20. Wilson MC, Hayward RS, Tunis SR, Bass EB, Guyatt G. Users' guides to the Medical Literature. VIII. How to use clinical practice guidelines. B. what are the recommendations and will they help you in caring for your patients? The Evidence-Based Medicine Working Group. JAMA 1995; 274: 1630-1632.

21. Hayward RS, Wilson MC, Tunis SR, Bass EB, Guyatt G. Users' guides to the medical literature. VIII. How to use clinical practice guidelines. A. Are the recommendations valid? The Evidence-Based Medicine Working Group. JAMA 1995; 274: 570-574.

22. Richardson WS, Detsky AS. Users' guides to the medical literature. VII. How to use a clinical decision analysis. B. What are the results and will they help me in caring for my patients? Evidence Based Medicine Working Group. JAMA 1995; 273: 1610-1613.

23. Richardson WS, Detsky AS. Users' guides to the medical literature. VII. How to use a clinical decision analysis. A. Are the results of the study valid? Evidence-Based Medicine Working Group. JAMA 1995; 273: 1292 1295.

24. Oxman AD, Cook DJ, Guyatt GH. Users' guides to the medical literature. VI. How to use an overview. Evidence-Based Medicine Working Group. JAMA 1994; 272: 1367-1371.

25. Laupacis A, Wells G, Richardson WS, Tugwell P. Users' guides to the medical literature. V. How to use an article about prognosis. Evidence-Based Medicine Working Group. JAMA 1994; 272: 234-237.

26. Levine M, Walter S, Lee H, Haines T, Holbrook A, Moyer V. Users' guides to the medical literature. IV. How to use an article about harm. Evidence-Based Medicine Working Group. JAMA 1994; 271: 16151619.

27. Jaeschke R, Guyatt GH, Sackett DL. Users' guides to the medical literature. III. How to use an article about a diagnostic test. B. What are the results and will they help me in caring for my patients? The EvidenceBased Medicine Working Group. JAMA 1994; 271: 703-707.

28. Jaeschke R, Guyatt G, Sackett DL. Users' guides to the medical literature. III. How to use an article about a diagnostic test. A. Are the results of the study valid? Evidence-Based Medicine Working Group. JAMA 1994; 271: 389-391.

29. Guyatt GH, Sackett DL, Cook DJ. Users' guides to the medical literature. II. How to use an article about therapy or prevention. B. What were the results and will they help me in caring for my patients? EvidenceBased Medicine Working Group. JAMA 1994; 271: 59-63.

30. Guyatt GH, Sackett DL, Cook DJ. Users' guides to the medical literature. II. How to use an article about therapy or prevention. A. Are the results of the study valid? Evidence-Based Medicine Working Group. JAMA 1993; 270: 2598-2601.

31. Oxman AD, Sackett DL, Guyatt GH. Users' guides to the medical literature. I. How to get started. The Evidence-Based Medicine Working Group. JAMA 1993; 270: 2093-2095. 
32. Begg C, Cho M, Eastwood S, Horton R, Moher D, Olkin I et al. Improving the quality of reporting of randomized controlled trials. The CONSORT statement. JAMA 1996; 276: 637-639.

33. Bossuyt PM, Reitsma JB, Bruns DE, Gatsonis CA, Glaszious PP, Irwig LM, et al. Towards complete and accurate reporting of studies of diagnostic accuracy: The STARD Initiative. Ann Intern Med 2003; 138: 40-44.

34. Manterola C, Pineda V, Vial M, Losada H, Muñoz S. Revisión sistemática de la literatura. Propuesta metodológica para su realización. Rev Chil Cir 2003; 55: 204-208.

35. Bornhöft G, Maxion-Bergemann S, Wolf U, Kienle GS, Michalsen A, Vollmar HC, et al. Checklist for the qualitative evaluation of clinical studies with particular focus on external validity and model validity. BMC Med Res Methodol 2006; 11: 56.

36. Fineout-Overholt E, Melnyk BM. Evaluation of studies of prognosis. Evid Based Nurs 2004; 7: 4-8.

37. Hayden JA, Côté P, Bombardier C. Evaluation of the quality of prognosis studies in systematic reviews. Ann Intern Med 2006; 144: 427-437.

38. Streiner DL, Norman GR. Reliability. Health Measurement Scales. Ontario, Canada: Oxford University Press, 1998: 104-126.

39. Polit DF, Hungler BP. Procedimientos estadísticos avanzados. Investigacion cientifica. Ciudad de México: McGraw Hill Interamericana, 1997: 435-475.

40. The Japanese Trial to Assess Optimal Systolic Blood Pressure in Elderly Hypertensive Patients (JATOS): Protocol, Patient Characteristics, and Blood Pressure during the First 12 Months. Hypertens Res 2005; 28: 513-520.

41. Abou-Khalil B, Schaich L. Long-term efficacy of levetiracetam for partial seizures. Seizure 2005; 14: 557-585.

42. Akaraviputh T, Boonnuch W, Lohsiriwat V, Methasate A, Chinswangwatanakul V, Lert-akayamanee N, et al. Long-Term Results of Large Diameter Hepaticojejunostomy for Treatment of Bile Duct Injuries Following Cholecystectomy. J Med Assoc Thai 2006; 89: 657-662.

43. Al-Ruzzeh S, Ambler G, Asimakopoulos G, Omar R, Hasan R, Fabri B, et al. Off-Pump Coronary Artery Bypass (OPCAB) Surgery Reduces Risk-Stratified Morbidity and Mortality: A United Kingdom MultiCenter Comparative Analysis of Early Clinical Outcome. Circulation 2003; 108: 1-8.

44. Atzema C, Mower W, Hoffman J, Holmes J, Killian A, Wolfson A. Prevalence and Prognosis of Traumatic Intraventricular Hemorrhage in Patients with Blunt Head Trauma. J Trauma 2006; 60:1010-1017.

45. Atar E, Bachar G, Eitan M, Graif F, Neyman H, Belenky A. Peripheral cutting balloon in the management of resistant benign ureteral and biliary strictures: long-term results. Diagnostic and Interven- tional Radiology 2007; 13: 39-41.

46. Ballard J, McFarland C, Silver L, Holiday D, Roberson G. The Effect of 15 Weeks of Exercise on Balance, Leg Strength, and Reduction in Falls in 40 Women Aged 65 to 89 Years. JAMWA 2004; 59: 255-261.

47. Bartsch R, Wenzel C, Pluschnig U, Hussian D, Sevelda $\mathrm{U}$, Altorjai $\mathrm{G}$, et al. Prognostic value of monitoring tumour markers CA 15-3 and CEA during fulvestrant treatment. BMC Cancer 2006; 6: 1-7.

48. Bastiaenen C, De Bie R, Wolters P, Vlaeyen J, Bastiaanssen J, Klabbers A, et al. Treatment of pregnancy-related pelvic girdle and/or low back pain after delivery design of a randomized clinical trial within a comprehensive prognostic cohort study. BMC Public Health 2004; 4: 1-12.

49. Becattini C, Agnelli G, Prandoni P, Silingardi M, Salvi $\mathrm{R}$, Taliani $\mathrm{M}$ et al. A Prospective study on cardiovascular events after acute pulmonary embolism. European Heart Journal 2005; 26: 77-83.

50. Beghi E, Tonini C. Surgery for epilepsy: Assessing evidence from observational studies. Epilepsy Research 2006; 1-6.

51. Bodí V, Facila L, Sanchis J, Llácer A, Núñez J, Mainar L, et al. Short-Term Prognosis of Patients Admitted for Probable Acute Coronary Syndrome without STSegment Elevation. Role of New Myocardial Damage Markers and Acute-Phase Reactants. Rev Esp Cardiol 2002; 55: 823-830.

52. Bohm M, Pronicka E, Karczmarewicz E, Pronicki M, Piekutowska-Abramczuk D, Sykut-Cegielska J et al. Retrospective, Multicentric Study of 180 Children with Cytochrome c Oxidase Deficiency. Pediatr Res 2006; 59: 21-26.

53. Bottio T, Rizzoli G, Caprili L, Nesseris G, Thiene G, Gerosa G. Full-Sternotomy Off-Pump versus On-Pump Coronary Artery Bypass Procedures. Tex Heart Inst J 2003; 30: 261-267.

54. Bradford N, Shappell S, Chang S, Wells N, Farnham S, Smith $\mathrm{J}$, et al. Tumour volume is an independent predictor of prostate-specific antigen recurrence in patients undergoing radical prostatectomy for clinically localized prostate cancer. Urological Oncology 2006; 97: 1169-1172.

55. Brouman B, Sadat M, Shafiel M. Impact of Hepatitis C Virus Infection on Short-Term Outcomes in Renal Transplantation. Experimental and Clinical Transplantation 2004; 2: 242-245.

56. Brunner M, Olschewski M, Geibel A, Bode C, Zehender M. Long-term survival after pacemaker implantation.Prognostic importance of gender and baseline patient characteristics. European Heart Journal 2004; 25: 88-95.

57. Budoff M, Shaw L, Liu S, Weinstein S, Mosler T, Tseng $\mathrm{P}$, et al. Long-Term Prognosis Associated With Coronary Calcification. J Am Coll Cardiol 2007; 49: 1860-1870. 
58. Calverley P, Boonsawat W, Cseke Z, Zhong N, Peterson S, Olsson H. Maintenance therapy with budesonide and formoterol in chronic obstructive pulmonary disease. Eur Respir J 2003; 22: 912-919.

59. Canavan C, Abrams K, Hawthorne B, Drossman D, Mayberry J. Long-term prognosis in Crohn's disease: factors that affect quality of life. Aliment Pharmacol Ther 2006; 23: 377-385.

60. Cappelleti V, Celio L, Bajetta E, Allevi A, Longarini $\mathrm{R}$, Miodini $\mathrm{P}$, et al. Prospective evaluation of estrogen receptor- $\beta$ in predicting response to neoadjuvant antiestrogen therapy in elderly breast cancer patients. Endocrine-Related Cancer 2004; 11: 761-770.

61. Catelin C, Tordjman S, Morin V, Oger E, Sizun J. Clinical, Physiologic, and Biologic Impact of Environmental and Behavioral Interventions in neonates During a Routine Nursing Procedure. The Journal of Pain 2006; 12: 791-797.

62. Chang-Kwon O, Sawyer R, Pelletier S, Pruett T, Sanfey H. Independent Predictors for Primary Non-Function after Liver Transplantation. Yonsei Medical Journal 2004; 45: 1155-1161.

63. Cheung A, Levin N, Greene T, Agodoa L, Bailey J, Beck $\mathrm{G}$ et al. Effects of High-Flux Hemodialysis on Clinical Outcomes: Results of the HEMO Study. J Am Soc Nephrol 2003; 14: 3251-3263.

64. Cheung C, Tsoi T, Hon S, Au-Yeung M, Shiu K, Lee C, et al. Outcomes after first-ever stroke. Hong Kong Med J 2007; 13: 95-99.

65. Chow T, Kereiakes D, Bartone C, Booth T, Schloss E, Waller T, et al. Prognostic Utility of Microvolt TWave Alternant in Risk Stratification of Patients With Ischemic Cardiomyopathy. J Am Coll Cardiol 2006; 47: $1820-1827$.

66. Colan S, McElhinney D, Crawford E, Keane J, Lock J. Validation and Re- Evaluation of a Discriminant Model Predicting Anatomic Suitability for Biventricular Repair in Neonates With Aortic Stenosis. J Am Coll Cardiol 2006; 47: 1858-1865.

67. De Waele J, Hoste E, Blot S, Hesse U, Pattyn P, De Hemptinne $\mathrm{B}$, et al. Perioperative factors determine outcome after surgery for severe acute pancreatitis. Critical Care 2004; 8: 504-511.

68. Dignam J, Wieand K, Johnson K, Fisher B, Xu L. Obesity, Tamoxifen Use, and Outcomes in Women With Estrogen Receptor-Positive Early-Stage Breast Cancer. J Natl Cancer Inst 2003; 95: 1467-1476.

69. Dome J, Cotton C, Perlman E, Breslow N, Kalapurakal J, Ritchey M, et al. Treatment of Anaplastic Histology Wilms' Tumor: Results From The Fifth National Wilms' Tumor Study. J Clin Oncol 2006; 24: $2352-$ 2358.

70. Dunkelgrun M, Hoeks S, Elhendy A, Van Domburg R, Bax J, Noordzij P, et al. Significance of hypotensive response during dobutamine stress echocardiography. International Journal of Cardiology 2007; 20: 1-6.
71. Elahi M, Hadjinikolaou L, Galiñanes M. Incidence and Clinical Consequences of Atrial Fibrillation Within 1 Year of First-Time Isolated Coronary Bypass Surgery. Circulation 2003; 108: 207-212.

72. Empana J, Ducimetiére P, Arveiler D, Ferriéres J, Evans A, Ruidavets J, et al. Are the Framingham and PROCAM coronary heart disease risk functions applicable to different European populations? European Heart Journal 2003; 24: 1903-1911.

73. Ewald N, Marzeion A, Bretzel R, Ulrich H, Hardt P. Endoscopic Sphincterotomy in patients with stenosis of ampulla of Vater: Three-year follow-up of exocrine pancreatic function and clinical symptoms. World $\mathrm{J}$ Gastroenterol 2007; 13: 901-905.

74. Fairburn C, Agras S, Walsh T, Wilson T, Stice E. Prediction of Outcome in Bulimia Nervosa by Early Change in Treatment. Am J Psychiatry 2004; 161: 2322-2324.

75. Fairman R, Nolte L, Snyder S, Chuter T, Greenberg R. Factors predictive of early or late aneurysm sac size change following endovascular repair. J Vasc Surg 2006; 43: 649-656.

76. Falvo L, Giacomelli Laura, D'Andrea V, Marzullo A, Guerriero G, De Antoni E. Prognostic Importance of Sclerosing Variant in Papillary Thyroid Carcinoma. The American Surgeon 2006; 72: 438-444.

77. Farinati F, Marino D, De Giorgio M, Baldan A, Cantarini M, Cursaro C, et al. Diagnostic and Prognostic Role of Fetoprotein in Hepatocellular Carcinoma: Both or Neither? Am J Gastroenterol 2006; 101: 524-532.

78. Fergusson D, Boden J, Horwood J. Abortion Among Young Women And Subsequent Life Outcomes. Perspectives on Sexual and Reproductive Health 2007; 39: 6-12.

79. Ferrer J, Domínguez A, García M, Abreu P, Bethencourt M, Marrero F. Influencia de la diabetes mellitus en el tratamiento y el pronóstico del síndrome coronario agudo sin elevación del segmento ST. Rev Esp Cardiol 2006; 59: 383-386.

80. Flori H, Glidden D, Rutherford G, Matthay M. Pediatric Acute Lung Injury Prospective Evaluation of Risk Factors Associated with Mortality. American Journal of Respiratory and Critical Care Medicine 2005; 171: 995-1001.

81. Franco E, Palumbo L, Crobu F, Anselmino M, Frea S, Matullo G, et al. Renin-angiotensin-aldosterone system polymorphisms: a role or a hole in ocurrence and long-term prognosis of acute myocardial infarction at young age. BMC Medical Genetics 2007; 8

82. Franneby U, Sandblom G, Nordin P, Nyrén O, Gunnarsson U. Risk Factors for Long-term Pain After Hernia Surgery. Ann Surg 2006; 244: 212-219.

83. Freixinet J, Juliá-Serdá G, Rodríguez P, Santana N, Rodríguez F, Fiuza M, et al. Hospital volume: operative morbidity, mortality and survival in thoracotomy for 
lung cancer. A Spanish multicenter study of 2994 cases. European Journal of Cardio-thoracic Surgery 2006; 29: $20-25$.

84. Furlan A, Sandoval J, Mailis-Gagnon A, Tunks E. Opioids for chronic noncancer pain: a meta-analysis of effectiveness and side effects. CMAJ 2006; 174: 1589-1594.

85. Garland A, Dawson N, Altmann I, Thomas C, Phillips R, Tsevat J, et al. For Pulmonologists, Cardiologists, Cardiothoracic Surgeons, Critical Care Physicians, and Related Specialists. CHEST 2004; 126: 1897-1904.

86. Gogas H, Ioannovich J, Dafni U, Stavropoulou-Giokas C, Frangia K, Tsoutsos D, et al. Prognostic Significance of Autoimmunity during Treatment of Melanoma with Interferon. N Engl J Med 2006; 354: 709-718.

87. González M. Resultado del tratamiento de la hemorragia subaracnoidea debida a rotura de aneurismas cerebrales. Neurocirugía 2006; 17: 433-439.

88. Halim A, Hamidon B, Cheong S, Raymond A. The prognostic value of tissue factor levels in acute ischaemic stroke. Singapore Med J 2006; 47: 400403.

89. Hayashi D, Yamazaki T. Design and Rationale of the Japanese Coronary Artery Disease (JCAD) Study. Jpn Heart J 2004; 45: 895-911.

90. Hee Kang J, Cook N, Manson J, Buring J, Grodstein F. Low dose aspirin and cognitive function in the women's health study cognitive cohort. BMJ 2007; 1-8.

91. Henkin Y, Shai I. Dietary Treatment of Hypercholestrolemia: Can We Predict Long-Term Success? Journal of the American College of Nutrition 2003; 22: $555-561$.

92. Herr H, Faulkner J, Barton H, Natale R, De Vere White R, Sarosdy M, et al. Surgical Factors Influence Bladder Cancer Outcomes: A Cooperative Group Report. J Clin Oncol 2004; 22: 2781-2789.

93. Ho Benjamin, Bellomo R, McGain F, Jones D, Naka $\mathrm{T}$, Wan $\mathrm{L}$, et al. The incidence and outcome of septic shock patients in the absence of early-goal directed therapy. Critical Care 2006; 10:1-7.

94. Hoye A, Iakovou I, Ge L, Van Mieghem C, Ong A, Cosgrave J, et al. Long-Term Outcomes After Stenting of Bifurcation Lesions With the "Crush" Technique. JACC 2006; 47: 1949-1958.

95. Huttunen R, Laine J, Lumio J, Vuento R, Syrjanen J. Obesity and smoking are factors associated with poor prognosis in patients with bacteraemia. BMC Infectious Diseases 2007; 7:1-8.

96. Huynh T, O'Loughlin J, Joseph L, Schampaert E, Rinfret S, Afilalo M, et al. Delays to reperfusion therapy in acute ST-segment elevation myocardial infarction: results from the AMI-QUEBEC Study. CMAJ 2006; 175: 1527-1532.

97. Ives A, Saunders C, Bulsara M, Semmens J. Pregnancy after breast cancer: population based study. BMJ 2007; 334: 194
98. Jensen D, Damm P, Moelsted-Pedersen L, Ovesen P, Westergaard J, Moeller M, et al. Outcomes in type 1 Diabetic Pregnancies. A nationwide, population-based study. Diabetes Care 2004; 27: 2819-2823.

99. Junginger T, Gockel I, Heckhoff S. A comparison of transhiatal and transthoracic resections on the prognosis in patients with squamous cell carcinoma of the esophagus. EJSO 2006; 1-7.

100. Kaji S, Akasaka T, Horibata Y, Nishigami K, Shono $\mathrm{H}$, Katayama $\mathrm{M}$, et al. Long-Term Prognosis of Patients With Type A Aortic Intramural Hematoma. Circulation 2002; 106: 248-252.

101. Kaliaperumal S, Rao V, Parija SC. Cysticercosis of the eye in south India- A case series. Indian Journal of Medical Microbiology 2005; 23: 227-230.

102. Kaposi-Novak P, Seog J, Gómez L, Coulouarn C, Factor $\mathrm{V}$, Thorgeirsson S. Met-regulated expression signature defines a subset of human hepatocellular carcinomas with poor prognosis and aggressive phenotype. J Clin Invest 2006; 116: 1582-1595.

103. Kestenbaum B, Sampson J, Rudser K, Patterson D, Seliger S, Young B. Serum Phosphate Levels and Mortality Risk among People with Chronic Kidney Disease. J Am Soc Nephrol 2005; 16: 520-528.

104. Kim J, Park S, Jeong J, Lee S, Gwon H, Kim K, et al. Clinical Features and Prognosis of Acute Aortic Intramural Hemorrhage Compared with Those of Acute Aortic Dissection. Jpn Heart J 2001; 42: 91-100.

105. Kitahara T, Takeishi Y, Arimoto T, Niizeki T, Koyama Y, Sasaki T, et al. Serum Carboxy-Terminal Telopeptide of Type I Collagen (ICTP) Predicts Cardiac Events in Chronic Heart Failure Patients With Preserved Left Ventricular Systolic Function. Circulation Journal 2007; 71: 929-935.

106. Ko D, Tu J, Masoudi F, Wang Y, Havranek E, Rathore S, et al. Quality of Care and outcomes of Older Patients With Heart Failure Hospitalized in the United States and Canada. Arch Inter Med. 2005; 165: 2486-2492.

107. Koenig W, Lowel H, Baumert J, Meisinger C. CReactive Protein Modulates Risk Prediction Based on the Framingham Score: Implications for Future Risk Assessment: Results From a Large Cohort Study in Southern Germany. Circulation 2004; 109: 1349-1353.

108. Korosec H, Jagodic K, Podbregar M. Long-Term outcome and quality of life of patients treated in surgical intensive care: a comparison between sepsis and trauma. Critical Care 2006; 10: 1-7.

109. Kurosaki H, Oriuchi N, Okazaki A, Tamaki T, Uki A, Izuta $\mathrm{M}$, et al. Prognostic value of FDG-PET in patients with ovarian carcinoma following surgical treatment. Annals of Nuclear Medicine 2006; 3: 171-174.

110. Lai Ch, Chi Ch, Chen H, Chen T, Lai Ch, Fung Ch, et al. Clinical characteristics and prognostic factors of patients with Stenotrophomonas maltophilia bacteremia. J Microbiol Immunol Infect 2004; 37: 350358. 
111. Langagergaard V, Gislum M, Skriver MV, Norgard B, Lash TL, Rothman KJ et al. Birth outcome in women with breast cancer. British Journal of Cancer 2006; 94: 142-146.

112. Le G, O'Malley C, Glaser S, Lynch C, Stanford J, Keegan $\mathrm{T}$, et al. Breast implants following mastectomy in women with early-stage breast cancer: prevalence and impact on survival. Breast Cancer Research 2005; 7: 184-193.

113. Leong K, Goh P, Chang B, Lingamanaicker J. Heart Failure cohort in Singapore with defined criteria: clinical characteristics and prognosis in a multi-ethnic hospital-based cohort in Singapore. Singapore Med J 2007; 48: 408-414.

114. Letho M, Snapinn S, Dickstein K, Swedberg K, Nieminen M. Prognostic risk of atrial fibrillation in acute myocardial infarction complicated by left ventricular dysfunction: the OPTIMAAL experience. European Heart Journal 2005; 26: 350-356.

115. Ljung T, Karlén P, Schmidt D, Hellstrom P, Lapidus A, Janczewska I, et al. Infliximab in inflammatory bowel disease:clinical outcome in a population based cohort from Stockholm Country. Gut 2004; 53: 849853.

116. Losada H, Manterola C, Pineda V, Vial M. Asociación entre recuento bacteriano en la bilis y variables fisiológicas con las complicaciones postquirúrgicas en pacientes con colangitis aguda en un hospital del sur de Chile. En prensa Rev Chil Cir.

117. Marín M, Hollier L, Ellozy S, Spielvogel D, Mitty H, Griepp R, et al. Endovascular Stent Graft Repair of Abdominal and Thoracic Aortic Aneurysms. Ann Surg 2003; 238: 586-595.

118. McCulloch P, Ward J, Tekkis P. Mortality and morbidity in gastro-oesophageal cancer surgery: initial results of ASCOT multicentre prospective cohort study. BMJ 2003; 327: 1192-1197.

119. McCulloch P, Ward J, Tekkis P. Mortality and morbidity in gastro-oesophageal cancer surgery: initial results of ASCOT multicentre prospective cohort study. BMJ 2006; 327: 1-6.

120. McDuffie C, Amirghahari N, Caldito G, Lian T, Thompson L, Nathan C. Predictive Factors for Posterior Triangle Metastasis in HNSCC. Laryngoscope 2005; 115: 2114-2117.

121. Meijer R, Van Limbeek J, Peusens G, Rulkens M, Dankoor K, Vermeulen M, et al. The Stroke unit Discharge Guideline, a prognostic Framework for the discharge outcome from the hospital stroke unit. A prospective cohort study. Clinical Rehabilitation 2005; 19: $770-778$.

122. Meisinger C, Loewel H, Mraz W, Koenig, W. Prognostic value of apolipoprotein B and A-I in the prediction of myocardial infarction in middle-aged men and women: results from the MONICA/KORA Augsburg cohort study. European Heart Journal 2005;
26: $271-278$.

123. Melloni G, Doglioni C, Bandiera A, Carretta A, Ciriaco P, Arrigoni G, et al. Prognostic Factors and Analysis of Microsatellite Instability in Resected Pulmonary Metastases From Colorectal Carcinoma. Ann Thorac Surg 2006; 81: 2008-2013.

124. Menéndez R, Nauffal D, Cremades M. Prognostic factors in restoration of pulmonary flow after submassive pulmonary embolism: a multiple regression analysis. Eur Respir J 1998; 11: 560-564.

125. Meyerhardt J, Catalano P, Schrag D, Ayanian J, Haller D, Mayer R, et al. Association of Hospital Procedure Volume and Outcomes in Patients with Colon Cancer at High Risk for Recurrence. Ann Intern Med 2003; 139: 649-657.

126. Miquel J, Abad R, Souto J, Fabra R, Vila M, Bargalló $\mathrm{D}$, et al. EUS-guided mucosectomy for gastrointestinal cancer. Revista Española de Enfermedades Digestivas 2006; 98: 591-596.

127. Morris M, Lacopetta B, Platell C. Comparing Survival outcomes for patients with colorectal cancer treated in public and private hospitals. MJA 2006; 186: 296300 .

128. Morris M, Lacopetta B, Platell C. Comparing survival outcomes for patients with colorectal cancer treated in public and private hospitals. MJA 2007; 186: 296300 .

129. Motohashi M. Profile of bilateral anterior cruciate ligament injuries: A retrospective follow-up study. Journal of Orthopaedic Surgery 2004; 12: 210-215.

130. Mukamal K, Maclure M, Muller J, Sherwood J, Mittleman M. Caffeinated coffee consumption and mortality after acute myocardial infarction. Am Heart J 2004; 147:999-1004.

131. Muller S, Prolla J, Maguilnik I, Breyer H. Predictive Factors of Oxygen Desaturation of Patients Submitted To Endoscopic Retrograde Cholagiopancreatography Under Conscious Sedation. Arq Gastroenterol 2004; 41: $162-166$.

132. Muneuchi G, Miyabe K, Hoshikawa H, Hata Y, Suzuki $\mathrm{S}$, Igawa $\mathrm{H}$, et al. Postoperative complications and long-term prognosis of microsurgical reconstruction after total maxillectomy. Microsurgery 2006; 26: 171176.

133. Panda S, Goyal V, Behari M, Singh S, Srivastava T. Myasthenic crisis: A retrospective study. Neurology India 2004; 52: 453-456.

134. Penido J, Santos J, Parizzoto V, Moreira E, Bahia L, Affonso M, et al. Clinical course of 735 children and adolescents with primary vesicoureteral reflux. IPNA 2006.

135. Pérez K, Titus-Ernstoff L, Hatch E, Troisi R, Wactawski-Wende J, Palmer J, et al. Reproductive outcomes in men with prenatal exposure to diethylstilbestrol. Fertil Steril 2005; 84: 1649-1656.

136. Pérez de Luque D, López P, Montero J, Fraga E, 
Barrera, P,Costán G et al. Survival of patients receiving a liver transplant for hepatocellular carcinoma, and risk of tumor recurrence. Revista Española de Enfermedades Digestivas 2006; 98: 899-906.

137. Pineda J, Marín F, Roldán V, Valencia J, Marco P, Sogorb F. Premature myocardial infarction: Clinical profile and angiographic findings. International Journal of Cardiology 2007; 20: 1-3.

138. Piombo A, Gagliardi J, Guetta J, Fuselli J, Salzberg S, Fairman E, et al. A new scoring system to stratify in unstable angina. BMC Cardiovascular Disorders 2003; 3:2-9.

139. Rajah J, Riera-Fanego J, Keeton J, Ramjee A, Bhana $\mathrm{R}$, Lasersohn L, et al. Do steroids prevent reintubation in children with laryngotracheobronchitis? Crit Care 2000; 4: 314-318.

140. Rech T, Rios S, Nagel F, Salles J, Scalco R. Serum neuron-specific enlace as early predictor of outcome after in-hospital cardiac arrest: a cohort study. Critical Care 2006; 10:1-6.

141. Rosted P, Bundgaard M. Can Acupuncture Reduce the Induction Time of a Local Anaesthetic? - A Pilot Study. Acupuncture in Medicine 2003; 21: 92-99.

142. Sacco S, Marini C, Totaro R, Russo T, Cerone D, Carolei A. A population-based study of the incidence and prognosis of lacunar stroke. Neurology 2006; 66: 1335-1338.

143. Sanchís J, Bertomeu V, Bodí V, Núñez J, Lauwers C, Ruiz-Nodar J, et al. Estrategia invasiva en pacientes con diabetes avanzada y síndrome coronario agudo sin elevación del segmento ST. Hallazgos angiográficos y evolución clínica. Resultados del estudio PREDICAR. Rev Esp Cardiol 2006; 59: 321-328.

144. Shishehbor M, Topol E, Mukherjee D, Hu T, Cohen D, Stone G et al. Outcome of Multivessel Coronary Intervention in the Contemporary Percutaneous Revascularization Era. Am J Cardiol 2006; 97: 15851590.

145. Singhi P, Bansal A, Geeta P, Singhi S. Predictors of Long Term Neurological Outcome in Bacterial Meningitis. Indian Journal of Pediatrics 2007; 74: 369374.

146. Sogaard O, Lemoh C, Spelman D, Freundlich M, Schonheyder H, Jensen R, et al. A binational cohort study of ventilator-associated pneumonia in Denmark and Australia. Scandinavian Journal of Infectious Diseases 2006; 38: 256-264.

147. Soto G, Jones P, Weintraub W, Krumholz H, Spertus J. Prognostic Value of Health Status in Patients With Heart Failure After Acute Myocardial Infarction. Circulation 2004; 110: 546-551.

148. Staresinic A, Sorkness C, Goodman B, Pigarelli D. Comparison of Outcomes Using 2 Delivery Models of Anticoagulation Care. Arch Intern Med. 2006; 166: 997-1002.

149. Stein P, Henry J, Relyea B. Untreated patients with pulmonary embolism. Outcome, clinical, and laboratory assessment. CHEST 1995; 107: 931-935.

150. Strate T, Taherpour Z, Bloechle C, Mann O, Bruhn J, Schneider C, et al. Long-term Follow-up of a Randomized Trial Comparing the Beger and Frey Procedures for Patients Suffering From Chronic Pancreatitis. Ann Surg 2005; 241: 591-598.

151. Tadokoro K, Miyamoto H, Sumi M, Shimomura. The Prognostic of Conservative Treatments for Lumbar Spinal Stenosis. Spine 2005; 30: 2458-2463.

152. Texereau J, Jamal D, Choukroun G, Burgel P, Diehl J, Rabbat A, et al. Determinants of mortality for adults with cystic fibrosis admitted in Intensive Care Unit: a multicenter study. Respiratory Research 2006; 7: 14.

153. Toh C, Wong E, Lim W, Leong S, Fong K, Wee J, et al. The Impact of Smoking Status on the Behavior and Survival Outcome of Patients With Advanced Non-small Cell Lung Cancer: A Retrospective Analysis. CHEST 2004; 126: 1750-1756.

154. Tuttolomondo A, Pedone C, Pinto A, Di Raimondo D, Fernández P, Di Sciacca R, et al. Predictors of outcome in acute ischemic cerebrovascular syndromes: The GIFA study. International Journal of Cardiology 2006; 20: 1-6.

155. Ural E, Yuksel H, Pehlivanoglu S, Bakay C, Olga R. Short and Long Term Survival of Surgical Treatment of Left Ventricular Aneurysms. Jpn Heart J 2002; 43: 379-387.

156. Van Balken M, Vergunst H, Bemelmans B. Prognostic Factors for Successful Percutaneous Tibial Nerve Stimulation. European Urology 2006; 49: 360-365.

157. Van de Beek D, De Gans J, Spanjaard L, Weisfelt M, Reitsma J, Vermeulen M. Clinical Features and Prognostic Factors in Adults with Bacterial Meningitis. N Engl J Med 2004; 351: 1849-1859.

158. Van Walraven C, Jennings A, Oake N, Fergusson D, Forster A. Effect of Study Setting on Anticoagulation Control. CHEST 2006; 129: 1155-1166.

159. Vukmir R, Katz L. Sodium bicarbonate improves outcome in prolonged prehospital cardiac arrest. American Journal of Emergency Medicine 2006; 24: 156-161.

160. Wapnir I, Anderson S, Mamounas E, Geyer Jr C, Jeong J, Tan-Chiu, et al. Prognostic After Ipsilateral Breast Tumor Recurrence and Locoregional Recurrences in Five National Surgical Adjuvant Breast and Bowel Project Node-Positive Adjuvant Breast Cancer Trials. J Clin Oncol 2006; 24: 2028-2037.

161. Ward J, Cherry J, Chang S, Partridge S, Lee H, Treanor $\mathrm{J}$, et al. Efficacy of an Acellular Pertussis Vaccine among Adolescents and Adults. N Engl J Med 2005; 353: 1555-1563.

162. Webster S, Lohse C, Thompson H, Dong H, Frigola $\mathrm{X}$, Dicks D, et al. Mononuclear Cell Infiltration in Clear-Cell Renal Cell Carcinoma Independently Predicts Patient Survival. Cancer 2006; 107: 46-53. 
163. Wilson I, Duszynski K, Mant A. A 5-year follow-up of general practice patients experiencing depression. Family Practice 2003; 20: 685-689.

164. Yoshida M, Ohtsu A, Boku N, Miyata Y, Shirao K, Shimada Y, et al. Long-term Survival and Prognostic Factors in Patients with Metastatic Gastric Cancers Treated with Chemotherapy in the Japan Clinical Oncology Group (JCOG) Study. Jpn J Clin Oncol 2004; 34: 654-659.

165.Zeymer U, Vogt A, Zahn R, Weber M, Tebbe U, Gottwik M, et al. Predictors of in-hospital mortality in 1333 patients with acute myocardial infarction complicated by cardiogenic shock treated with primary percutaneous coronary intervention (PCI). European Heart Journal 2004; 25: 322-328.

166.Zheng L, Jia H, Wu D, Shang H, Zhong X, Wang Q, et al. Experience of Congenital Choledochal Cyst in Adults: Treatment, Surgical Procedures and Clinical Outcome in the Second Affiliated Hospital of Harbin
Medical University. J Korean Med Sci 2004; 19: 842847.

167. Zhou L, Rui J, Wang S, Chen S, Qu Q, Chi T, et al. Clinicopathological features, post-surgical survival and prognostic indicators of elderly patients with hepatocellular carcinoma. EJSO 2006; 20: 1-6.

168. Zhou P, Chen M, McLeod D, Carroll P, Moul J, D'Amico A. Predictors of Prostate Cancer-Specific Mortality After Radical Prostatectomy or Radiation Therapy. J Clin Oncol 2005; 23: 6992-6998.

169. Dixon E, Hameed M, Sutherland F, Cook D, Doig C. Evaluating meta-analyses in the general surgical literature. A critical appraisal. Ann Surg 2005; 241: 450-459.

170. Moher D, Tetzlaff J, Tricco A, Sampson M, Altman D. Epidemiology and reporting characteristics of systematic reviews. PLoS Medicine 2007; 4: 447-455. Disponible en: http:www.medicine.plosjournals.org. Consultado octubre de 2007. 